\title{
Controle Preditivo Baseado em Modelo para Conversores Formadores de Rede com Operação Ilhada
}

\author{
Rayane Aparecida Guimarães*, Silvia Costa Ferreira, \\ Vinicius Miranda Pacheco, João Paulo de Carvalho Pedroso, \\ João Eduardo Ribeiro, Overlan Silva Viana, \\ Daniel Furtado Leite, \\ * Departamento de Automática,Universidade Federal de Lavras, MG \\ (e-mail: rayane.ap@outlook.com; silvia.ferreira@ufla.br).
}

\begin{abstract}
Microgrids composes several distributed generation, storage and load systems. However, controllers and systems are complex, especially in island mode operation. In this condition, the grid forming converters are responsible for the proper functioning of the microgrid, controlling voltage amplitude and frequency according to given references. There are several control tecniques to operate a grid forming converter, one of then is the model based predictive control, which predicts the future behavior of the system in order to define the switching state which best minimizes a cost function. This work consists in the design of a grid forming power converter for island mode operation, and also in de development of an model based predictive control algorithm to operate this converter. The developed system was simulated in software (MATLAB/Simulink) under diverse load conditions and with voltage reference and load variations for validating its operation. The results obtained shows that the proposed model is capable of controling the voltage from a given reference value under diverse load patterns, maintaining the voltage signal even after its reference value are changed.

Resumo: Uma microrrede é composta por vários sistemas de geração distribuída, armazenamento e cargas. No entanto, o controle e operação desses sistemas são complexos, principalmente quando operam em modo ilhado. Nessa condição, os conversores formadores de rede são responsáveis por manterem o funcionamento adequado da microrrede, controlando a amplitude da tensão e a frequência de acordo com uma dada referência. Existem várias técnicas de controle para operar um conversor, uma delas é o controle preditivo baseado em modelo, o qual prevê o comportamento futuro do sistema e escolhe o melhor estado de chaveamento dos semicondutores de acordo com a minimização de uma função custo. Esse trabalho consiste em projetar um conversor formador de rede para operar em modo ilhado e desenvolver um algoritmo de controle preditivo baseado em modelo para atuar nesse conversor. $\mathrm{O}$ sistema desenvolvido foi simulado em software MATLAB/Simulink para diversos tipos de cargas e variações nas referências de tensão e carga, com o objetivo de validar seu funcionamento. Os resultados obtidos mostram que o sistema proposto realiza a regulação de tensão a partir da referência para diversos tipos de cargas e mantém o sinal de tensão após o valor de referência sofrer alterações.
\end{abstract}

Keywords: Finite Control Set Model Predictive Control, Grid-Forming Converters, Microgrid. Palavras-chaves: Controle Preditivo Baseado em Modelo, Conversor Formador de Rede, Microrredes.

\section{INTRODUÇÃO}

Uma microrrede pode ser definida como um sistema que engloba unidades de geração distribuída, sistemas de armazenamento de energia e cargas locais. Esse sistema deve entregar energia confiável e de qualidade. Além disso, pode operar em dois modos: conectado à rede elétrica ou de forma ilhado (Ferreira et al., 2018).

$\mathrm{Na}$ maioria das vezes, as fontes renováveis de energia dependem de situações climáticas e ambientais de comportamento estocástico, sendo imprevisíveis em curtos espaços de tempo. Isso torna esse tipo de geração instável para pequenos intervalos sendo necessária a utilização de conversores para controlar e gerenciar o fluxo de potência. Segundo Patrao et al. (2017), os conversores eletrônicos utilizados em microrredes podem ser classificados em três grupos: conversor alimentador de rede (CAR), de suporte à rede $(\mathrm{CSR})$ e o formador de rede $(\mathrm{CFR})$.

$\mathrm{O}$ CAR é projetado para fornecer energia ativa à rede elétrica. O CSR ajusta sua corrente e a tensão de saída para ajudar na regulação desses parâmetros da rede para que se aproximem de seus valores nominais. Já o CFR é utilizado para definir a amplitude da tensão e a frequência 
da rede local utilizando uma malha de controle adequada (Rocabert et al., 2012).

Ao longo dos anos foram elaboradas diversas técnicas de controle aplicadas aos conversores de potência. Alguns desses métodos de controle são: fuzzy, adaptativos, modo deslizante e preditivos (MPC). O método tradicional mais utilizado é o controlador linear PI (Proporcional-Integral) no sistema de referência síncrona. Porém, esse tipo de controle vem perdendo espaço, principalmente quando o sistema a ser controlado possui restrições e não linearidades (Kouro et al., 2009).

No entanto, o controle preditivo baseado em modelo com conjunto de controle finito (FCS-MPC - Finite Control Set Model Predictive Control) é capaz de lidar com tais situações. Sendo adequado para várias aplicações, principalmente em circuitos de eletrônica de potência (Cortes et al., 2008).

Este trabalho consiste em desenvolver e projetar um CFR alimentado por baterias operando em modo ilhado, utilizando o FCS-MPC para regular a tensão desse conversor. O principal objetivo é testar a capacidade do algoritmo em seguir uma determinada referência de tensão, rejeitando os distúrbios das cargas conectadas ao sistema. Para validar o funcionamento desse sistema desenvolvido, o CFR monofásico, com potência nominal de $2 \mathrm{~kW}$ é simulado no software MATLAB/Simulink, onde também é testado o algoritmo de controle do FCS-MPC sob diferentes condições de cargas, lineares e não lineares, e com alterações nos valores de referência. Ao realizar esses testes observouse que o sistema proposto foi capaz de seguir a referência independente das variações de cargas testados. Permitindo assim que esse sistema seja implementado na prática futuramente.

\section{CONVERSOR FORMADOR DE REDE - CFR}

A Figura 1 apresenta um CFR monofásico com a topologia em ponte $\mathrm{H}$, o qual tem sido amplamente empregado em fontes de energias renováveis (Queiroz et al., 2017). A alimentação desse conversor ocorre por meio de um banco de baterias no barramento CC. Na saída da Ponte $\mathrm{H}$ existe um transformador para elevar a tensão de saída do conversor $\left(v_{i}\right)$ para o valor CA desejado.

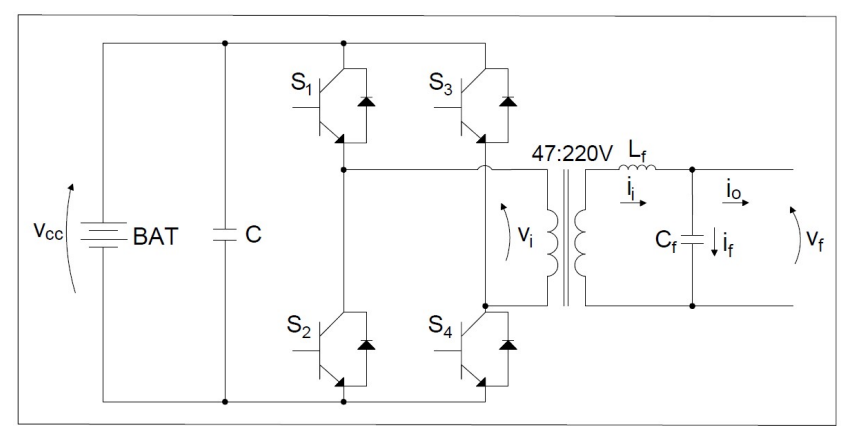

Figura 1. Topologia do CFR com transformador e filtro LC.

Para filtrar os harmônicos de alta frequência, resultantes do chaveamento de conversores eletrônicos, são utilizados filtros passivos do tipo LC em busca de uma tensão de alta qualidade (Kahlane et al., 2014). O filtro LC projetado para essa aplicação utiliza de uma indutância $\left(L_{F}\right)$ e uma capacitância $\left(C_{F}\right)$. A tensão de entrada do filtro é representada por $\left(v_{i}\right)$ e a tensão de saída por $\left(v_{f}\right)$, a qual é a mesma aplicada a carga.

Existem vários métodos para controlar o chaveamento dos semicondutores. O mais utilizado na literatura são os controladores PI baseados em referência síncrona. No entanto, Rodriguez et al. (2013) compararam esse tipo de controle utilizando modulação PWM com o MPC (Model Predictive Control), chegando a conclusão que o MPC é comparável às soluções de controle clássicas, sendo superior em termos de desempenho e flexibilidade. Os autores relataram que as vantagens do MPC são: a eliminação da cascata de controladores, a facilidade de lidar com as não linearidades e a capacidade de incluir restrições, tais como algoritmos para eliminar ressonâncias causadas pelos filtros de saída.

\section{CONTROLE PREDITIVO BASEADO EM MODELO - MPC}

Em termos gerais o MPC (Model Predictive Control) pode ser considerado como qualquer algoritmo que usa um modelo do sistema para prever seu comportamento futuro e selecionar a ação de controle mais apropriada com base em um critério de otimização (Kouro et al., 2009). Esse tipo de controle é bastante aceito por ser aplicado em vários tipos de sistema e pela possibilidade de considerar as restrições e não linearidades do sistema. Outro fato relevante, é que o MPC é intuitivo e de fácil implementação. Por outro lado, esse método requer um grande número de cálculos quando comparado às formas de controle clássicas.

A técnica de controle preditivo baseada em modelo pode ser classificada em duas categorias principais: conjunto de controle contínuo (CCS-MPC - Continuous Control Set Model Predictive Control) e conjunto de controle finito (FCS-MPC - Finite Control Set Model Predictive Control).

No CCS-MPC um modulador gera os estados de comutação a partir da saída contínua do controlador preditivo. Por outro lado, o FCS-MPC aproveita a natureza discreta dos conversores para reduzir os cálculos e o tempo de processamento do MPC. Como há um número finito de comutações das chaves de um conversor, o procedimento de previsão será limitado apenas a esses estados, sendo um destes selecionado devido ao processo de minimização dado pela função custo. Os principais elementos deste esquema de controle são o modelo matemático do sistema e a função custo predefinida (Cortes et al., 2008; Kouro et al., 2009).

No entanto, a frequência de chaveamento e a velocidade do microprocessador devem ser levadas em consideração para aplicações deste tipo de controle, uma vez que, pode ocorrer um atraso entre o tempo de medição da variável de controle e o tempo de aplicação do estado de chaveamento escolhido. Para levar em consideração o tempo de processamento, o algoritmo de controle deve ser projetado de acordo com os seguintes passos:

(1) Medir a variável de controle;

(2) Aplicar o estado de chaveamento calculado no período de amostragem anterior;

(3) Estimar a variável de controle para o instante $k+1$; 
(4) Prever a variável de controle para o instante $k+2$ para todos os estados de chaveamento do conversor;

(5) Avaliar a função custo;

(6) Escolher o estado de chaveamento que minimiza a função custo o qual será aplicado no próximo período de amostragem.

\section{APLICAÇÃO DO FCS-MPC EM CONVERSORES FORMADORES DE REDE}

Para implementar o FCS-MPC, o modelo do sistema deverá ser derivado e discretizado de acordo com a variável a ser controlada, sendo essa a tensão de saída para o CFR (Innocent et al., 2018).

\subsection{Modelo Discreto do Sistema}

A partir da topologia do conversor apresentada na Figura 1 é possível encontrar o modelo discreto do sistema. Porém, devido as características do transformador, seus parâmetros devem ser considerados no modelo. Ferreira et al. (2018) modela o transformador considerando apenas a sua impedância série. Os autores justificam que após resultados práticos com FCS-MPC é possível desconsiderar o ramo magnetizante no modelo devido a sua baixa influência. Sendo assim, as impedâncias série do transformador são incluídas no modelo do conversor, como pode ser observado na Figura 2. Nesse caso, os parâmetros somam-se ao filtro LC, ou seja, a indutância do conversor (L) é igual ao somatório a indutância do transformador $\left(L_{t}\right)$ com a do filtro $\left(L_{f}\right), L=L_{t}+L_{f}$.



Figura 2. Topologia do CFR com filtro LC equivalente.

Com essa topologia é possível encontrar o modelo discreto do sistema a partir da tensão do conversor $\left(v_{i}\right)$, tensão no filtro $\left(v_{f}\right)$ a qual é a mesma aplicada na carga $\left(v_{o}\right)$, corrente do conversor $\left(i_{i}\right)$ e a correto do filtro $\left(i_{f}\right)$.

$$
\begin{gathered}
L \frac{d i_{i}(t)}{d t}=v_{i}(t)-v_{f}(t)-R i_{i}(t) \\
C_{f} \frac{d v_{f}(t)}{d t}=i_{i}(t)-i_{o}(t)
\end{gathered}
$$

Para representar esse modelo em um sistema em espaço de estados é considerado que $i_{i}(t)$ e $v_{f}(t)$ são as variáveis de estado e $v_{i}(t)$ e $v_{o}(t)$ são as de controle. Posteriormente, o sistema é discretizado utilizando Euler Forward como apresentado em Panten et al. (2016), resultando nas seguintes equações:

$$
\begin{array}{r}
{\left[\begin{array}{c}
i_{i}(k+1) \\
v_{f}(k+1)
\end{array}\right]=\left[\begin{array}{cc}
\left(1-\frac{T_{s} R}{L}\right) & \frac{-T_{s}}{L} \\
\frac{T_{s}}{C_{f}} & 1
\end{array}\right]\left[\begin{array}{c}
i_{i}(k) \\
v_{f}(k)
\end{array}\right]+} \\
{\left[\begin{array}{cc}
\frac{T_{s}}{L} & 0 \\
0 & \frac{-T_{s}}{C_{f}}
\end{array}\right]\left[\begin{array}{c}
v_{i}(k) \\
i_{o}(k)
\end{array}\right]}
\end{array}
$$

\subsection{Definição dos estados de chaveamento}

Para a topologia utilizada neste trabalho (Figura 1) as chaves $S_{1}$ e $S_{2}$ e as chaves $S_{3}$ e $S_{4}$ são comutadas de maneira complementar. Sendo assim, a Tabela 1 apresenta os quatro estados de comutação dos semicondutores da ponte $\mathrm{H}$, onde os vetores de comutação estão em função da tensão do barramento CC $\left(V_{C C}\right)$.

Tabela 1. Estados de chaveamento para a topologia da Figura 1.

\begin{tabular}{ccccc} 
S1 & S2 & S3 & S4 & Saída \\
\hline 0 & 1 & 0 & 1 & 0 \\
1 & 0 & 0 & 1 & $V_{C C}$ \\
0 & 1 & 1 & 0 & $-V_{C C}$ \\
1 & 0 & 1 & 0 & 0 \\
\hline
\end{tabular}

\subsection{Definição da função custo}

A função custo é responsável por minimizar o erro entre a variável de controle e a de referência, escolhendo assim o melhor estado de chaveamento. Nessa aplicação, a variável a ser controlada é a tensão de saída do filtro $\left(v_{f}\right)$. Logo, a função custo pode ser observada na equação (4), onde $v_{f}^{*}$ é o valor de referência e $v_{f}^{p}$ é o valor previsto a partir do modelo discreto do sistema (Cortes et al., 2008).

$$
J=\left(v_{f}^{*}-v_{f}^{p}\right)^{2}
$$

\section{DESCRIÇÃO DO SISTEMA}

A simulação desse sistema foi realizada na plataforma MATLAB/Simulink utilizando a linguagem de programação C no bloco $s$-function para implementar o FCS-MPC. Os testes foram elaborados com cargas do tipo resistiva indutiva, resistiva capacitiva e não linear, com o objetivo de analisar a resposta do sistema frente a essas cargas, observando o THD (Total Harmonic Distortion) da tensão e da corrente de saída. Também foram elaborados testes para analisar a resposta do sistema em situações de variação da referência de tensão e de carga.

O diagrama de blocos da Figura 3 apresenta a sequência de funcionamento do CFR com FCS-MPC (Cortes et al., 2009). O estado de chaveamento $(S)$ para os semicondutores é obtido a partir das decisões tomadas pelo algoritmo de previsão implementado no bloco s-function. Esse bloco recebe a corrente de entrada do filtro $\left(i_{i}\right)$, a tensão filtrada $\left(v_{f}\right)$ e a tensão de referência $\left(v_{f}^{*}\right)$. As saídas do bloco correspondem aos estados de chaveamento $S_{1}, S_{2}, S_{3}$ e $S_{4}$.

As equações do modelo do sistema são inseridas no bloco $s$-function para que a predição possa ser feita. A Figura 4 


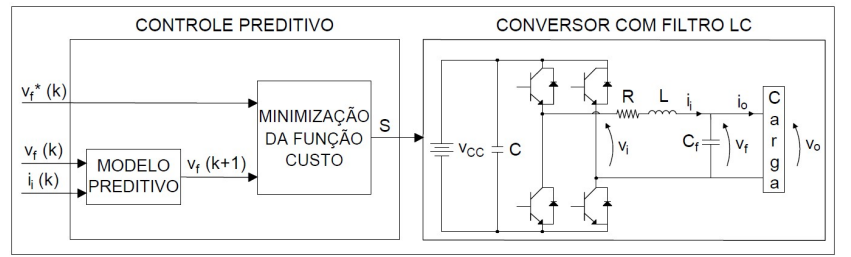

Figura 3. Diagrama do CFR com FCS-MPC.

apresenta o fluxograma de funcionamento do algoritmo do FCS-MPC. Como pode ser observado, o primeiro passo é medir as variáveis de estado $i_{i}(k)$ e $v_{f}(k)$ e o estado de chaveamento ótimo $\left(S_{o t}\right)$ é aplicado no instante $k$. A partir desse ponto o FCS-MPC estima $k+1$ com os valores medidos no sistema. Então, o algoritmo prevê as variáveis de estado para o instante $k+2$ para os três estados de chaveamento possíveis. Para cada estado o sistema avalia a função custo e verifica se essa foi minimizada. Os estados de chaveamento que resultaram nessa minimização são aplicados no conversor.

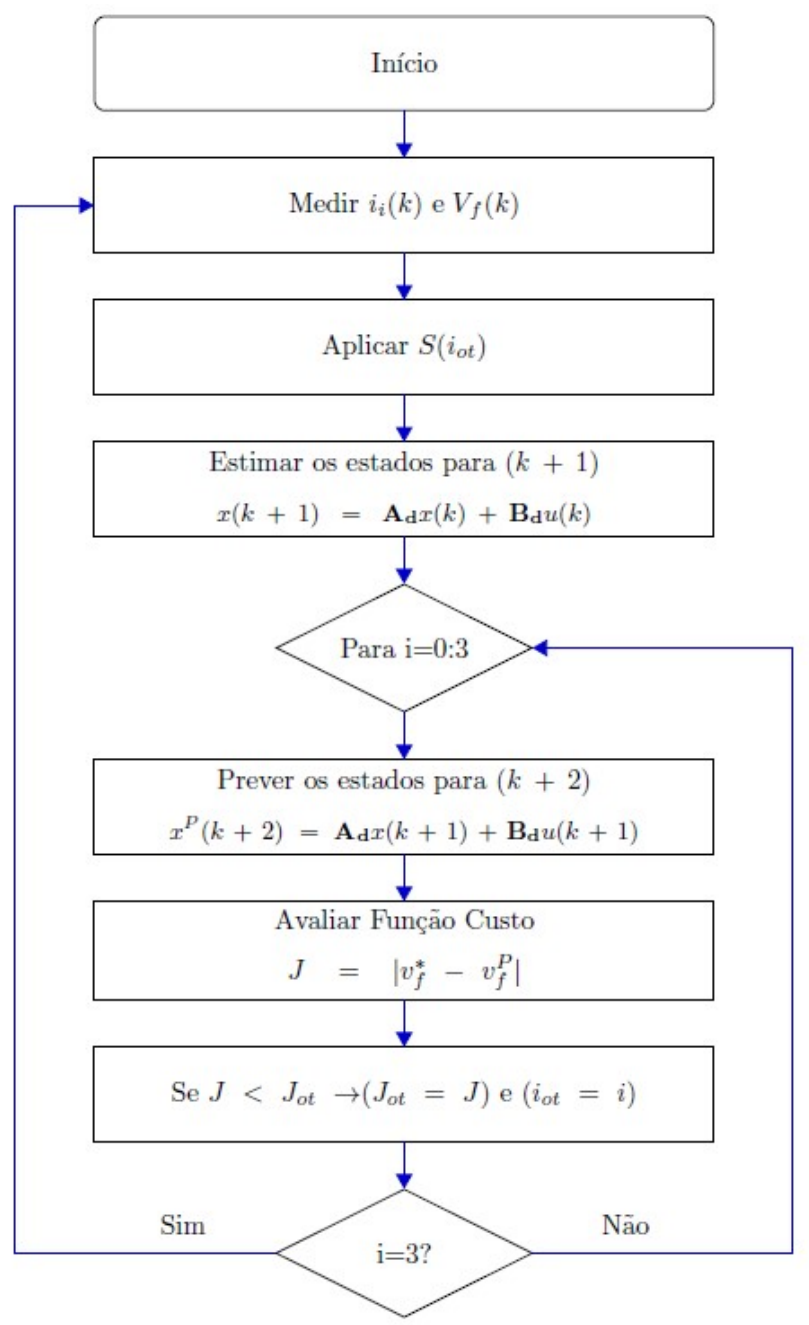

Figura 4. Fluxograma do FCS-MPC.

Com o objetivo de validar a operação do CFR com FCS-MPC sistema proposto foi simulado operando com diferentes tipos de cargas, além de variações de carga e na referência de tensão. Os parâmetros utilizados são apresentados na Tabela 2.
A seguir são apresentados os resultados e considerações para cada caso simulado.

Tabela 2. Parâmetros utilizados na simulação.

\begin{tabular}{ccc} 
Parâmetros & Variável & Valor \\
\hline Tensão de Referência & $v_{f}^{*}$ & $220 \mathrm{~V}$ \\
Frequência de Referência & $f$ & $60 \mathrm{~Hz}$ \\
Tensão das Baterias & $V_{c c}$ & $96 \mathrm{~V}$ \\
Capacitor CC & $C$ & $1000 \mu \mathrm{F}$ \\
Potência Transformador & $S$ & $2 \mathrm{kVA}$ \\
Relação de Transformação & & $47: 220 \mathrm{~V}$ \\
Resistência do Transformador & $R$ & $0,5 \Omega$ \\
Indutância do Equivalente & $L$ & $6,7 \mathrm{mH}$ \\
Indutância do Transformador & $L_{t}$ & $1,7 \mathrm{mH}$ \\
Indutância do Filtro & $L_{F}$ & $5,0 \mathrm{mH}$ \\
Capacitor do Filtro & $C_{F}$ & $10,5 \mu \mathrm{F}$ \\
Frequência de amostragem & $f_{a}$ & $40,08 \mathrm{kHz}$ \\
\hline
\end{tabular}

\subsection{Caso 1 - Carga $R L$}

Nessa simulação o conversor alimenta uma carga RL com potência ativa de $1 \mathrm{~kW}$ e indutiva de $1 \mathrm{kVAr}$. A Figura 5 apresenta a tensão $v_{f}$ gerada pelo CFR, a tensão de referência $\left(v_{f}^{*}\right)$ e a corrente da carga $i_{o}$.

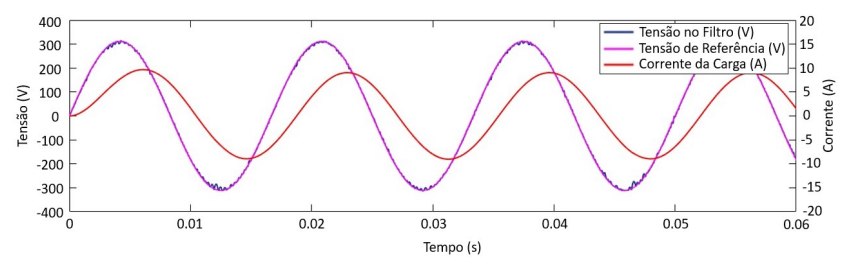

Figura 5. Tensão de referência $\left(v_{f}^{*}\right)$, tensão do filtro $\left(v_{f}\right)$ e corrente da carga $\left(i_{o}\right)$ para o caso 1.

Observa-se que o CFR conseguiu seguir a referência dada ao sistema e manter a regulação de tensão em torno de $311 \mathrm{~V}$ de pico. Além disso, o THD da corrente $(0,22 \%)$ é menor que o da tensão $(1,69 \%)$, devido as características indutivas da carga.

\subsection{Caso 2 - Carga RC}

Nessa etapa a carga RL é substituída por uma carga RC com $1 \mathrm{~kW}$ de potência ativa e $1 \mathrm{kVar}$ de potência reativa capacitiva. Assim como no caso anterior, nota-se que a resposta do sistema também foi satisfatória, onde a tensão permanece em aproximadamente $311 \mathrm{~V}$ de pico (Figura 6). O THD da tensão $(0,99 \%)$ é menor do que o da corrente $(1,40 \%)$.

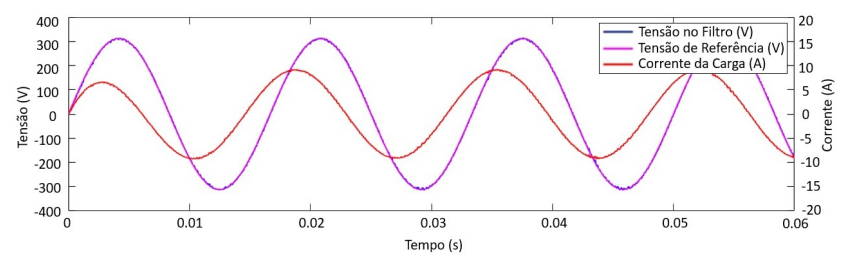

Figura 6. Tensão de referência $\left(v_{f}^{*}\right)$, tensão do filtro $\left(v_{f}\right)$ e corrente da carga $\left(i_{o}\right)$ para o caso 2.

Para todos os testes utilizando cargas lineares o CFR operando com FCS-MPC responde de forma satisfatória, com baixas distorções harmônicas e boa regulação de tensão. A seguir são apresentados os resultados para a carga não linear. 


\subsection{Caso 3 - Carga não linear}

Nesse caso o CFR alimenta uma carga não linear, representada por um retificador conectado a uma carga RL, com resistência de $26 \Omega$ e indutância de $100 \mathrm{mH}$, com potência aproximada de $1,5 \mathrm{~kW}$. Sabe-se que, torna-se difícil manter a tensão puramente senoidal quando uma carga não linear é conectada ao conversor, devido aos harmônicos presentes na corrente. A circulação de componentes harmônicos de corrente podem causar ressonâncias e aumentar o THD da tensão. Porém, o sistema proposto alcança um resultado satisfatório, regulando a tensão em $311 \mathrm{~V}$ de pico e mantendo-a com baixa distorção, como pode ser observado na Figura 7.



Figura 7. Tensão de referência $\left(v_{f}^{*}\right)$, tensão do filtro $\left(v_{f}\right)$ e corrente da carga $\left(i_{o}\right)$ para o caso 3 .

Analisando o THD é possível notar um aumento no THD da tensão para $2,11 \%$. Já o THD da corrente apresenta um valor de $39,6 \%$, devido as características não lineares da carga. Portanto, o FCS-MPC é capaz de manter uma tensão senoidal mesmo alimentando uma carga altamente distorcida.

\subsection{Caso 4 - Degrau na referência de tensão}

Nesse caso, o degrau de referência foi aplicado com o CFR alimentando uma carga RL com 500W de potência ativa e 500Var de potência reativa indutiva. O degrau aplicado foi de $220 \mathrm{~V}$ para $127 \mathrm{~V}$ e ocorreu em um instante aleatório.

Como pode ser observado na Figura 8 a oscilação na tensão $v_{f}$ é referente ao tempo de acomodação, ocorre devido ao tempo em que o algoritmo de controle leva para se estabilizar, ou seja, buscar novos resultados a partir da nova referência. Nessa simulação esse tempo foi de aproximadamente $1,84 \mathrm{~ms}$. Nota-se ainda que mesmo alterando a referência, o FCS-MPC foi capaz de acompanhar a mudança e seguir a nova referência com tempo de acomodação muito baixo.

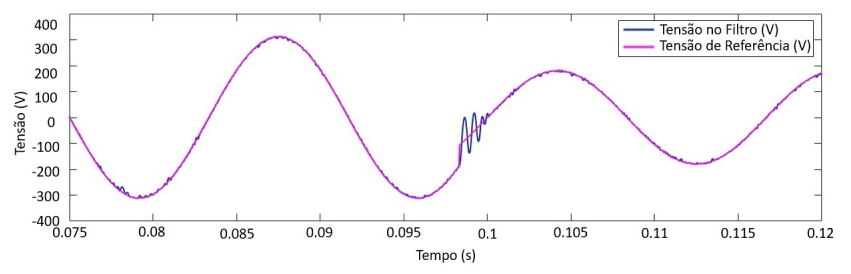

Figura 8. Tensão de referência $\left(v_{f}^{*}\right)$ e tensão do filtro $\left(v_{f}\right)$ para o caso 4.

\subsection{Caso 5 - Variação de Carga}

Ainda com a carga RL conectada ao CFR com tensão de referência de $220 \mathrm{~V}$ é simulado uma variação de carga, inserindo em paralelo uma carga RL idêntica a existente. A Figura 9 apresenta os sinais de tensão de referência $\left(v_{f}^{*}\right)$, tensão no filtro $\left(v_{f}\right)$ e a corrente na carga $\left(i_{o}\right)$. Observa-se que mesmo com a variação de carga a tensão $v_{f}$ não foi afetada. Assim como nos geradores síncronos, o controle preditivo consegue atuar como um regulador de tensão. Nesse sistema, a corrente foi aumentada e a tensão não sofreu nenhuma alteração devido a mudança na carga.

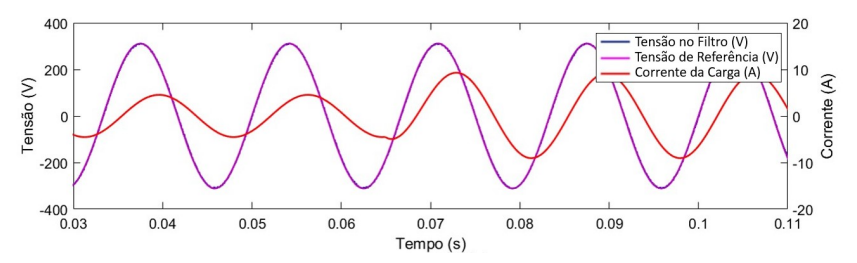

Figura 9. Tensão de referência $\left(v_{f}^{*}\right)$, tensão do filtro $\left(v_{f}\right)$ e corrente da carga $\left(i_{o}\right)$ para o caso 5.

\section{CONCLUSÕES}

Esse trabalho apresentou a modelagem, projeto e simulação de um CFR operando em modo ilhado com FCS-MPC. Sendo validado a partir de simulações com vários tipos de cargas e com variação de carga e aplicação de degrau na referência de tensão.

Em todas as simulações elaboradas os resultados mostraram que o sistema foi eficiente ao conseguir seguir a referência, não importando o tipo de carga e sua variação. Além disso, como pode ser observado no Caso 4, além de seguir a referência dada, o sistema respondeu de forma rápida ao degrau na referência de tensão.

Pode-se observar a partir dos resultados simulados, que a capacidade de rejeição de distúrbios foi muito boa para todos os tipos de cargas, apresentando THD máximo de $2,11 \%$ quando as cargas alimentadas são não lineares. Se comparado com técnicas tradicionais da literatura, esses resultados validam o FCS-MPC como um controle promissor.

Como etapas futuras serão dimensionados componentes para construir um CFR, com o objetivo de validar na prática o funcionamento do conversor proposto nesse trabalho.

\section{AGRADECIMENTOS}

Agradeço a UFLA, ao Departamento de Automática, ao Programa de Pós-Graduação em Engenharia de Sistemas e Automação, a CAPES e a FAPEMIG pelo financiamento.

\section{REFERÊNCIAS}

Cortes, P., Kazmierkowski, M.P., Kennel, R.M., Quevedo, D.E., and Rodriguez, J. (2008). Predictive control in power electronics and drives. IEEE Transactions on Industrial Electronics, 55(12), 4312-4324. doi:10.1109/ TIE.2008.2007480.

Cortes, P., Ortiz, G., Yuz, J.I., Rodriguez, J., Vazquez, S., and Franquelo, L.G. (2009). Model predictive control of an inverter with output lc filter for ups applications. IEEE Transactions on Industrial Electronics, 56(6), 1875-1883. doi:10.1109/TIE.2009.2015750. 
Ferreira, S., Gonzatti, R., Pereira, R., da Silva, C., da Silva, L., and Lambert-Torres, G. (2018). Finite control set model predictive control for dynamic reactive power compensation with hybrid active power filters. IEEE Transactions on Industrial Electronics, 2608-2617.

Innocent, L.A., Lamine, D.M., Joseph, S.M., and Yaramasu, V. (2018). Finite control set-model predictive control for power converters multilevel symmetric cascaded h-bridge inverters-fed drive. In 2018 Thirteenth International Conference on Ecological Vehicles and Renewable Energies (EVER), 1-7. doi:10.1109/EVER. 2018.8362412.

Kahlane, A., Hassaine, L., and Kherchi, M. (2014). Lcl filter design for photovoltaic grid connected systems. Revue des Energies Renouvelables SIENR14 Ghardaïa.

Kouro, S., Cortes, P., Vargas, R., Ammann, U., and Rodriguez, J. (2009). Model predictive control a simple and powerful method to control power converters. IEEE Transactions on Industrial Electronics, 56(6), 18261838. doi:10.1109/TIE.2008.2008349.

Panten, N., Hoffmann, N., and Fuchs, F.W. (2016). Finite control set model predictive current control for gridconnected voltage-source converters with lcl filters: A study based on different state feedbacks. IEEE Transactions on Power Electronics, 31(7), 5189-5200. doi: 10.1109/TPEL.2015.2478862.

Patrao, I., González-Medina, R., Marzal, S., Garcerá, G., and Figueres, E. (2017). Synchronization of power inverters in islanded microgrids using an fm-modulated signal. IEEE Transactions on Smart Grid, 8(1), 503510. doi:10.1109/TSG.2016.2574038.

Queiroz, A.D.P.D., Jacobina, C.B., de Freitas, N.B., Maia, A.C.N., and Melo, V.F.M.B. (2017). Single-phase ac-dcac multilevel converter based on h-bridges and three-leg converters connected in series. In 2017 IEEE Energy Conversion Congress and Exposition (ECCE), 21292136. doi:10.1109/ECCE.2017.8096421.

Rocabert, J., Luna, A., Blaabjerg, F., and Rodríguez, P. (2012). Control of power converters in ac microgrids. IEEE Transactions on Power Electronics, 27(11), 47344749. doi:10.1109/TPEL.2012.2199334.

Rodriguez, J., Kazmierkowski, M.P., Espinoza, J.R., Zanchetta, P., Abu-Rub, H., Young, H.A., and Rojas, C.A. (2013). State of the art of finite control set model predictive control in power electronics. IEEE Transactions on Industrial Informatics, 9(2), 1003-1016. doi: 10.1109/TII.2012.2221469. 